\title{
High volume microinfusion suppresses local astrocyte response within nucleus basalis of rat
}

\author{
I. BJELOBABA, J. SAPONJIC \\ University of Belgrade, Department of Neurobiology, \\ Institute for Biological Research, Belgrade, Serbia
}

\author{
A B S T R A C T
}

Our study investigates the impact of different volume sham control and excitotoxin microinfusions in vivo on local reactive astroglial response within rat nucleus basalis (NB). We followed the effects of unilateral 200, 100, and 50 $n L$ of sham-control (phosphate buffer PBS) versus ibotenic acid (IBO) microinfusions, mechanical NB lesion (1 $\mu L$ Hamilton syringe needle positioned into NB for $5 \mathrm{~min}$ ), or physiological control (intact brain), on the local reactive astroglial response within the NB site, by immunoreactivity against glial fibrillary acidic protein $(G F A P)$. NB lesions were identified by NADPH-diaphorase histochemistry. Local astrocytes responses within NB were suppressed by both high volume microinfusions, PBS and IBO (200 and $100 \mathrm{~nL}$ ) versus mechanical lesion. Our study has proved, for the first time, the volume of microinfusion as critical for any selective pharmacological stimulation or lesion in vivo, and suggest the microinfusion volume less than $50 \mathrm{~nL}$ as protective for physiological astroglial reactivity.

Key words

Astrogliosis $\bullet$ Ibotenic acid lesion $\bullet$ Glial fibrillary acidic protein $\bullet$ Nucleus basalis $\bullet$ Rat

\section{Introduction}

Astroglial response is very important in many physiological and pathological processes of the central nervous system (Bignami, 1995), and is detectable by usual immunohistochemical method against the glial fibrillary acidic protein (GFAP) (Eng and Ghirnikar, 1994; Monzon-Mayor et al., 2000; Swarowsky et al., 2008). Following brain injury the hypertrophy/GFAP hyper-reactivity has been observed not only at the lesion site (local response), but also in the remote locations where the lesion specific afferents project and target (MonzonMayor et al., 2000; Swarowsky et al., 2008). There is also evidence that astrocyte response is dependent on the specific neurons damaged within the brain. For instance, the cortical astrocytes changes have been observed after lesion of the cholinergic neurons of nucleus basalis, but not after dopaminergic injury in the ventral tegmental area (Anezaki et al., 1992). Prominent reactive astroglial response also followed the neuronal involution that characterized pathological processes of the neurodegenerative disorders. Alzheimer's disease (AD), the most common cause of dementia in the elderly, presents the 'dramatic' glial reactivity along with the typical AD lesions such as amyloid deposition, tangle formation, and loss/dysfunction of the cholinergic neurons of basal forebrain (Monzon-Mayor et al., 2000). Moreover, many years ago, a close relationship between amyloid deposits, normal and reactive glial cells was observed, and it has been suggested that glial dysfunction or response induces, collaborates with, or amplifies the amyloid precursor protein mismetabolism that produces amyloid deposits and neuronal damage. However, the correlation between cholinergic neuronal involution and reactive astrocytosis is poorly described, and is mostly 
considered as a secondary event. Lesions of the nucleus basalis magnocellularis (NB) in vivo have been widely used as an animal model for understanding the role of cholinergic neurotransmission in the central nervous system functions, and the disorders caused by cortical cholinergic neuronal degeneration such as Alzheimer's disease (Arendash et al., 1987; Anezaki et al., 1992; Monzon-Mayor et al., 2000; Toledano and Alvarez 2004, 2010; Swarowsky et al., 2008; Topchiy et al., 2010). Local stereotaxically guided nanovolume microinfusion/ microinjection technique in vivo enable us to pharmacologically stimulate, lesion, trace, and generally to investigate in animals the functional topography of certain brain nucleus with a resolution of $\leq 10$ $\mu \mathrm{m}$, and at a level of $300 \mu \mathrm{m}$ of effective radius within the brain tissue (Toledano and Alvarez 2004, 2010; McKay et al., 2005; Saponjic et al., 2003, 2005a, 2005b; Lu et al., 2006; Swarowsky et al., 2008; Topchiy et al., 2010). The advantage of local stimulation by glutamate or DL homocysteic acid microinjection, or local lesion by microinfusion of the excitotoxins (glutamate, ibotenic acid (IBO), IgG saporin), over electrical stimulation/lesion of the same neuronal population, is that they reduce the likelihood of activation/lesion of fibers of passage (Monnier et al., 2003). The mostly used and effective excitotoxin for unselective neuronal cell bodies lesion, and also for the lesion of NB in rat is IBO (Rugg et al., 1992; Inglis and Semba, 1997; Monzon-Mayor et al., 2000; Swarowsky et al., 2008). However, the appropriate microinfusion volume should be considered for the selective 'recruitment' of the certain neuronal population within the brain and also for the selective excitotoxic lesion in vivo of NB in rat, as the most utilized animal model of $\mathrm{AD}$ neuropathology. In this study, in order to determine the appropriate volume of IBO microinfusion for the complete lesion of the NB cholinergic neurons, we followed the local astrocyte response (microinfusion site at the tip of Hamilton syringe needle within NB) by immunoreactivity against glial fibrillary acidic protein (GFAP). We preformed the different volumes of sham-control (0.1 M PBS) and excitotoxin (0.1 M IBO/0.1 M PBS) microinfusions, and compared the astocyte response with respect to mechanical NB lesion (Hamilton syringe $1 \mu \mathrm{L}$ needle positioned into NB for $5 \mathrm{~min}$ ), or physiological control (intact brain).

\section{Methods}

\section{Animals and surgery}

We performed the experiments in 24 , adult (2 months old), male Wistar rats. Prior to surgery and consistently throughout the experimental protocol, animals were maintained on a 12-hour lightdark cycle, and were housed at $25^{\circ} \mathrm{C}$ with free access to food and water. Principles for the care and use of laboratory animals in research were strictly followed according to the Animal Care Committee of the Institute for Biological Research, University of Belgrade, Serbia, and conformed to recommendations given in the 'Guide for Care and Use of Laboratory animals' (National Academy of Sciences Press, Washington, DC, 1996). We lesioned NB unilaterally (right NB nucleus) under ketamine/diazepam anesthesia (50 $\mathrm{mg} \mathrm{kg}^{-1}$; i.p), using Digital Lab Standard ${ }^{\mathrm{TM}}$ Stereotaxic Instrument with Quintessential Stereotaxic Injector (Stoelting Europe), and Hamilton syringe ( $1 \mu \mathrm{l}, 26$ ga needle). We preformed the microinfusions of $50 \mathrm{~nL}, 100 \mathrm{~nL}$, or $200 \mathrm{~nL}$, in one $60 \mathrm{~s}$ pulse, of the $0.1 \mathrm{M}$ PBS (pH $=7.4$ ), as the unilateral sham control microinfusions $(\mathrm{n}=9)$, or $0.1 \mathrm{M} \mathrm{IBO} / 0.1 \mathrm{M}$ PBS (Sigma; $\mathrm{pH}=7.4$ ) for the unilateral NB lesions $(\mathrm{n}=9$; A/P: 1.4 from bregma; R: 3.0; D/V: 7.0) according to Paxinos and Watson (2004). Following stereotaxic positioning, and before removal from the brain, the Hamilton syringe was left within the local brain tissue for 5 min, to serve as a mechanical lesion control $(n=3)$, or to allow the microinfused solution to diffuse, and to recruit selectively a whole NB cholinergic neuronal subpopulation. Schematic summary diagram of the experimental design is presented on Fig. 1.

\section{Tissue processing and histochemistry}

After survival period of 7 days, the rats were deeply anesthetized and perfused intracardially, started with the vascular rinse until the liver had been cleared (200 ml; perfusion speed of $40 \mathrm{ml} / \mathrm{min}$ ); continued with a $4 \%$ paraformaldehyde solution in $0.1 \mathrm{M}$ phosphate buffer $(200 \mathrm{ml} ; 100 \mathrm{ml}$ at $40 \mathrm{ml} / \mathrm{min}$, and then $30 \mathrm{ml} / \mathrm{min}$ ), and with the $10 \%$ sucrose solution in $0.1 \mathrm{M}$ PBS (200 $\mathrm{ml} ; 30 \mathrm{ml} / \mathrm{min})$. The animals were sacrificed and the brains were extracted, cleared of the meninges and blood vessels, and immersed in $4 \%$ paraformaldehyde overnight, and then in $30 \%$ sucrose solution for several days. The brains were 


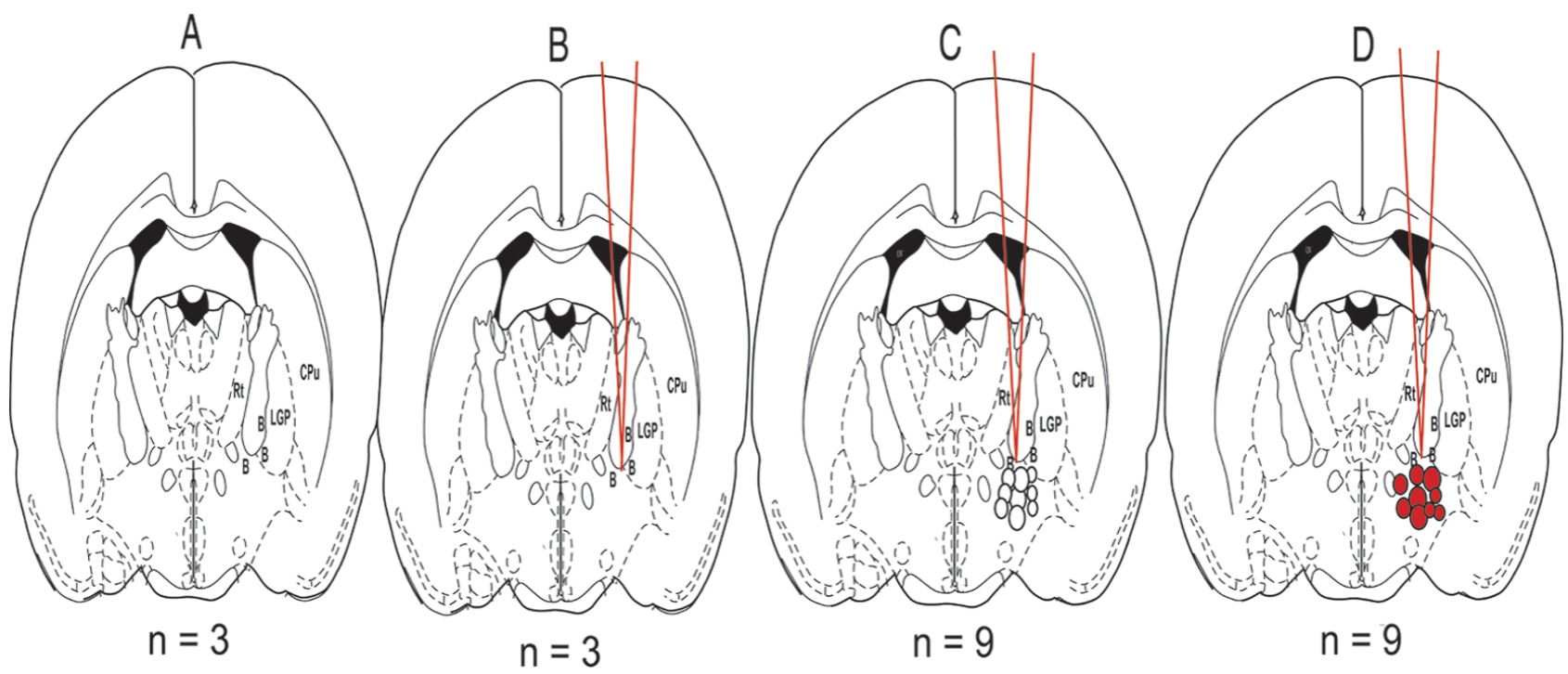

Fig. 1. - Schematic summary diagram of the experimental design: A: physiological control $(n=3)$; B: unilateral mechanical lesion within NB ( $n=3$; mechanical lesion control; A/P: 1.4 from bregma; R: 3.0; D/V: 7.0); C: the unilateral sham control microinfusions of the 0.1 M PBS (the size of empty circles corresponds to $200 \mathrm{~nL}, 100 \mathrm{~nL}$, and $50 \mathrm{~nL}$ of 0.1 M PBS ( $n=9$; A/P: 1.4 from bregma; R: 3.0; D/V: 7.0); $D$ : the unilateral microinfusions of 0.1 M IBO/0.1 M PBS ( $n$ = 9; A/P: 1.4 from bregma; R: 3.0; D/V: 7.0; the size of red (filled) circles corresponds to $200 \mathrm{~nL}, 100 \mathrm{~nL}, \mathrm{and} 50 \mathrm{~nL}$ ).

cut in a transverse plane in the $40 \mu \mathrm{m}$-thick sections using cryotome. Lesion of NB was identified by NADPH (diaphorase histochemical procedure) (Vincent et al., 1983; Dawson et al., 1991; Hope et al., 1991; Sabbatini et al., 1999; Datta et al., 2001). Briefly, the free floating sections were rinsed in 0.1 $\mathrm{M}$ PBS pH 7.4, and incubated one hour at $37^{\circ} \mathrm{C}$ in the staining solution-mixture of the substrate solution with $\beta$-nicotinamide adenine dinucleotide phosphate, reduced-Na4 salt ( $\beta$-NADPH, Serva), and dimethyl sulfoxide (DMSO, Sigma). Substrate solution contains dissolved nitro blue tetrazolium choride (NBT, Serva), and 5bromo-4chloro-3indolyl phosphate (BCIP, Serva) in the substrate buffer with pH 9.5 (0.1 M Tris $100 \mathrm{mM} \mathrm{NaCl}, 5 \mathrm{mM} \mathrm{MgCl} 2)$, To reduce the background staining coming from endogenous alkaline phosphatase, specific inhibitor levamisole (Sigma) was added to staining solution to a final concentration of $2 \mathrm{mM}$.

\section{Immunohistochemistry}

Astrocytes were identified by immunoperoxidase staining with rabbit polyclonal antibody for glial fibrilar acid protein (GFAP), following the standard procedure. Briefly, free-floating sections were rinsed several times in 0.01 M PBS pH 7.4. After neutralizing the endogenous peroxidase, non-specific bind- ing was reduced with $3 \%$ normal donkey serum in PBS. Primary antibodies to GFAP (DAKO, 1:1000 dilution) were applied overnight at $4{ }^{\circ} \mathrm{C}$. Horseradish peroxidase conjugated secondary donkey anti-rabbit antibody (Santa Cruz Biotechnology, Santa Cruz, CA) was used and the reaction product was visualized with 3'3-diaminobenzidine (DAB, Dako), according to manufacturer instructions. Sections were then mounted on the gelatine coated slides, coverslipped with DPX and examined under Zeiss Axiovert microscope equipped with camera.

We compared the astroglial response caused by different volume sham control (PBS; $n=9)$ or excitotoxin (IBO; $\mathrm{n}=9$ ) microinfusions with respect to GFAP immunoreactivity in physiological control brain $(n=3)$, and to unilateral mechanical lesion control $(\mathrm{n}=3)$.

\section{Results}

Our results have shown the diverse local astrocytes responses (at the microinfusion needle tip site within $\mathrm{NB})$ to the different volumes of unilateral sham-control (0.1 M PBS, Fig. 2C, E, G), and IBO microinfusions (Fig. 2D, F, H), with respect to physiological control NB site (intact brain; Fig. 2A), and unilateral 

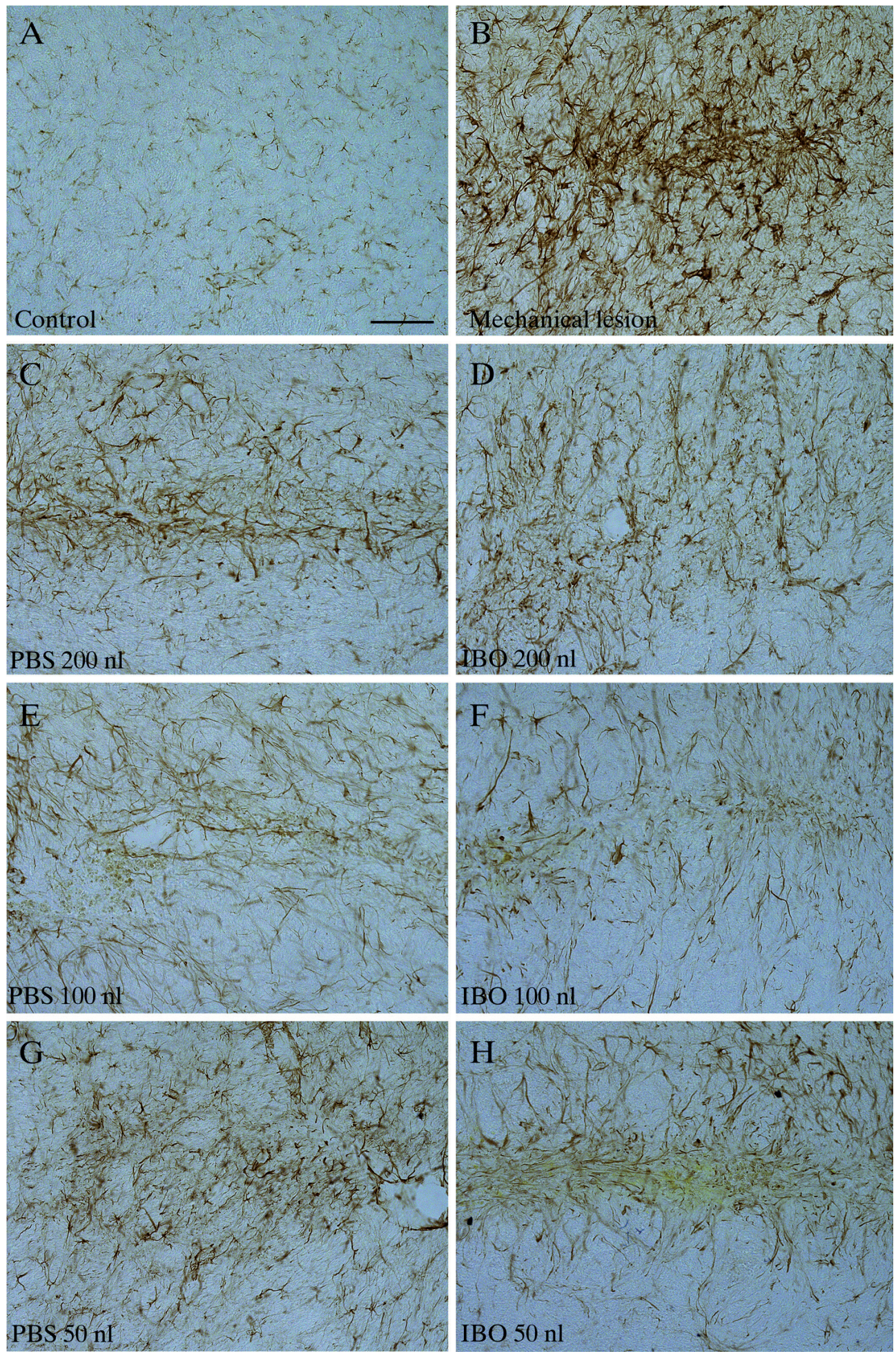

Fig. 2. - Local astrocyte responses (the needle tip site within NB) to different volumes of the sham-control (0.1 M PBS; $C, E, G)$, and IBO microinfusions (D, F, H) with respect to control brain (A) and unilateral mechanical lesion control (B) following survival time of 7 days. While the highly reactive astrocytes with gliofibril hyperproduction were present in the unilateral mechanical lesion (B), the higher volume unilateral sham controls (C - $200 \mathrm{~nL}$; and E - $100 \mathrm{~nL}$ ), and the unilateral NB lesion effective IBO microinfusions (D - $200 \mathrm{~nL}$; and F - $100 \mathrm{~nL}$ ) were characterized by decreased number of reactive astrocytes with respect to mechanical lesion control (B). Morphologically, these astrocytes had small bodies and long, thin processes resembling to fibrous type. Following $50 \mathrm{~nL}$ of PBS the reactive astrocytes occurred again (G), while following $50 \mathrm{~nL}$ IBO microinfusion the suppressed astrocytes response is still maintained (H). Scale bar in A applies to all images: $100 \mu \mathrm{m}$. 
mechanical lesion control (Fig. 2B; an effect of the stereotaxically positioned Hamilton syringe $1 \mu \mathrm{L}$ needle into NB for 5 min in situ). While the highly reactive astrocytes with gliofibril hyperproduction were present in the mechanical lesion control (Fig. 2B), the higher volume sham control (Fig. 2C - 200 nL 0.1 M PBS; Fig. 2E - 100 nL 0.1 M PBS), and the NB lesion effective IBO microinfusions (Fig. 2D - 200 nL 0.1 M IBO/0.1 M PBS; and Fig. 2F - 100 $\mathrm{nL} 0.1 \mathrm{M} \mathrm{IBO} / 0.1 \mathrm{M}$ PBS) were characterized by decreased number of the reactive astrocytes versus mechanical control 7 days after the microinfusions. Morphologically these astrocytes had small bodies and long, thin processes resembling to fibrous type. Following 7 days after the lower volume sham control microinfusion (Fig. 2G - $50 \mathrm{~nL} 0.1 \mathrm{M}$ PBS) the reactive astrocytes occurred again, in contrast to lower volume IBO microinfusion (Fig. 2H - 50 $\mathrm{nL}$ 0.1 M IBO/0.1 M PBS), where excitotoxin still suppressed astrocyte response. Still, this lower volume sham control microinfusion induced astroglial response was suppressed versus mechanical control. Although the astrocyte response was suppressed at the needle tip site within NB following the high volume sham-control microinfusions versus mechanical controls, the astrocyte response was equal within the cortex of the mechanical lesion controls and sham-controls. Fig. 3 depicts typical cortical (Fig. 3A - mechanical control; Fig. 3C - $200 \mathrm{~nL}$ sham-control) versus $\mathrm{NB}$ (Fig. 3B - mechanical control; Fig. 3D - $200 \mathrm{~nL}$ shamcontrol) astroglial response in mechanical control and $200 \mathrm{~nL}$ sham-control brain. Fig. 4 depicts an example of typical stereotaxic position of our microinfusion within NB stained by NADPH-diaphorase staining for "tracking" the cholinergic NB neurons (Fig. 4A, B), and the identified successful unilateral (right) excitotoxic NB lesion using $100 \mathrm{~nL}$ of $0.1 \mathrm{M} \mathrm{IBO/0.1} \mathrm{M} \mathrm{PBS}$ (Fig. 4B, D) versus control unlesioned NB side of the same brain (Fig. 4A, C). We achieved the selectivity and completeness of the NB lesion with $200 \mathrm{~nL}$ and $100 \mathrm{~nL}$ of IBO microinfusions, while $50 \mathrm{~nL}$ microinfusions failed to induce a complete NB lesion.

\section{Discussion}

We here compared the effects of different IBO and sham control microinfusion volumes on astrocyte response locally within NB. We have found that both high volume IBO and sham control microinfusions have profound effect on astrocyte reactivity, when compared to mechanical lesion control 7 days after the microinfusions. It is well known that IBO as excitotoxin impairs astrocyte response. Astrocytes grafted immediately after infusion of IBO into NB fail to survive (Fulop et al., 1997), and the hippocampal astrocytes show decrease in glutamate uptake, even 28 days after NB lesion with IBO (Swarowsky et al., 2008).

However, our data indicate that in addition to local and unselective effect of excitotoxin IBO on neuronal and glial cells within NB, the astrocytes responses were affected (suppressed) by the pressure within the local tissue of the high volume microinfusions of PBS and IBO (200 and $100 \mathrm{~nL}$ ). Although 7 days following microinfusion of $50 \mathrm{~nL}$ PBS (sham-control microinfusion) the reactive astrocytes occurred again, in contrast to microinfusion of $50 \mathrm{~nL}$ of IBO, this astroglial response is still suppressed versus mechanical control. However, the cortical astroglial response, due to positioned microinfusion needle, was equal in the sham-controls and mechanical controls. Although there is no much evidence in vivo, the Nicholson's theoretical calculation (Nicholson, 1985) predicts that the selectivity of pharmacological stimulation/lesion of any brain nucleus, or its neuronal subpopulation, is more dependent of the microinfusion/microinjection volume than the concentration of the microinjected/microinfused drug or solution. Nicholson's mathematical model predicts that there is no difference in diffusion of the same concentration of microinjected/microinfused solution between $1 \mathrm{~nL}$ and $10 \mathrm{~nL}$, but the diffusion distance within the brain tissue in vivo is changed significantly with an increase of the volume between $10 \mathrm{~nL}$ and $100 \mathrm{~nL}$. This study also predicts that $30 \mathrm{~s}$ following microinjection/microinfusion, the concentration of the drug present $300 \mu \mathrm{m}$ from the injection site would be no more than $20 \%$ of the concentration at the needle or pipette tip. Our in vivo study, using the equimolar concentration of excitotoxin $(0.1 \mathrm{M}$ IBO/0.1 M PBS), and the same survival time for all microinfusions (7 days), have proved for the first time, through astrocytes reactivity, the volume of microinfusion as critical for any selective pharmacological stimulation or lesion within a brain. It should be emphasized here that many experimental studies using NB lesion as the rat model of human 

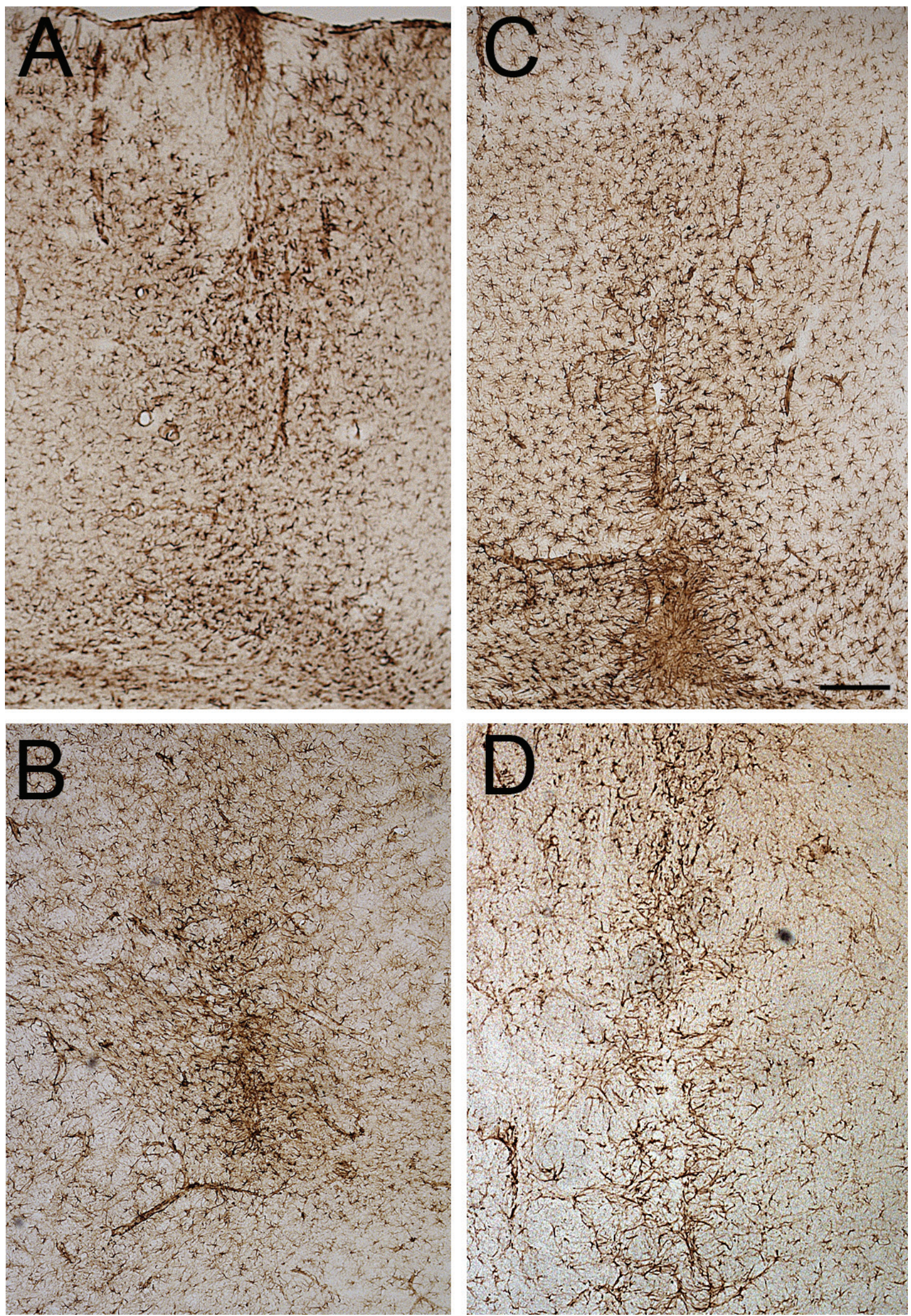

Fig. 3. - Typical example of cortical versus NB astrocytes response in mechanical control versus the highest volume sham-control microinfusion. Astroglial response within cortex of mechanical control (A), and following 200 $\mathrm{nL}$ sham-control microinfusion (C). Astroglial response within NB of mechanical control (B), and following $200 \mathrm{~nL}$ sham-control microinfusion (D). Scale bar $200 \mu \mathrm{m}$ applies to all images. 

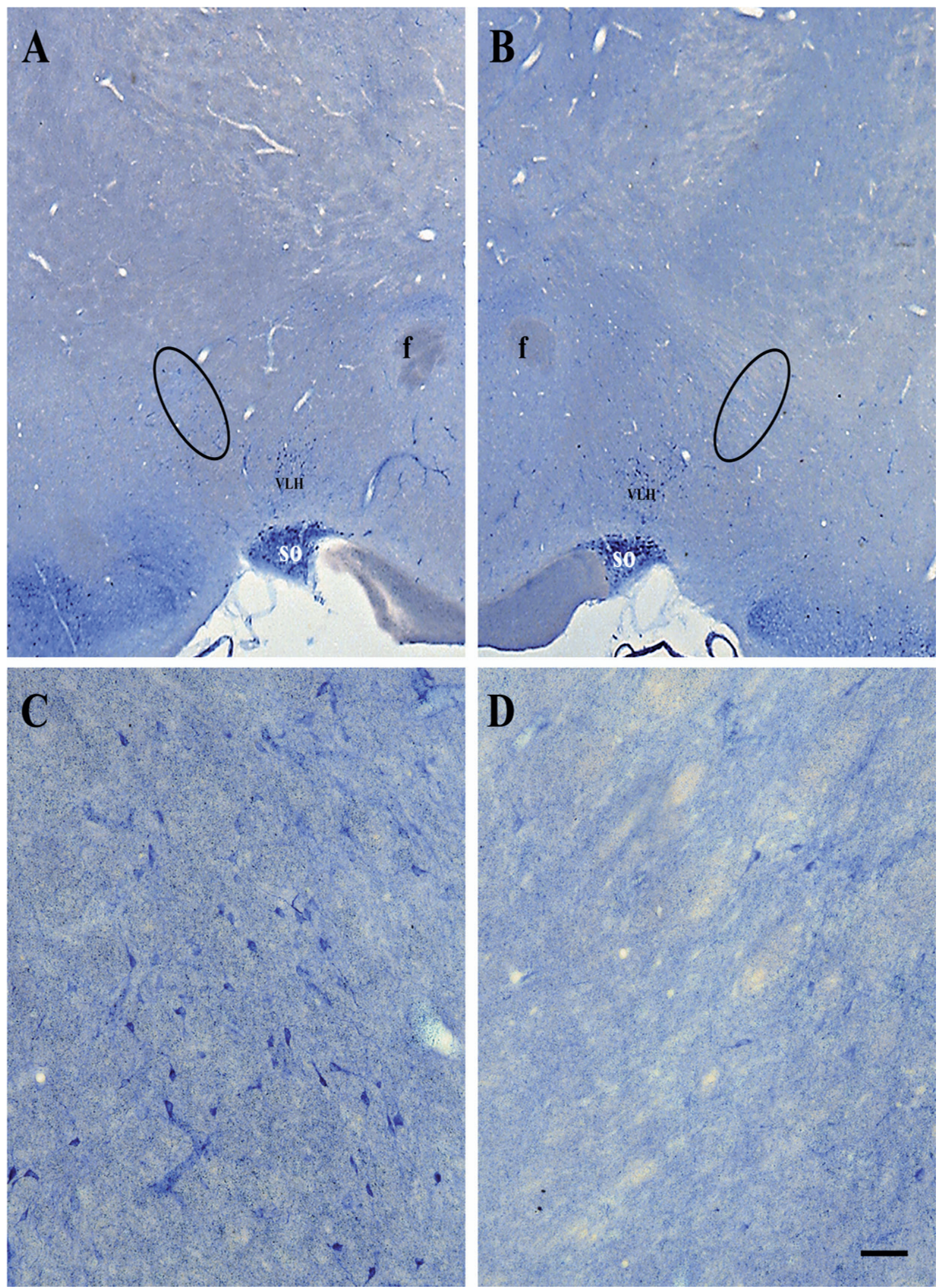

Fig. 4. - Typical stereotaxic position of the microinfusioins within NB (A/P: 1.4 from bregma; R: 3.0; D/V: 7.0): stained by NADPH-diaphorase staining (A, B, C, D), and its cholinergic neurons magnified (C, D). Number of the cholinergic neurons was decreased following unilateral microinfusion of $100 \mathrm{~nL}$ IBO into right NB (D), compared to control, left NB (C). Scale bar in C applies also to D: $50 \mu \mathrm{m}$. 
AD neuropathology (Arendash et al., 1987; Anezaki et al., 1992; Monzon-Mayor et al., 2000; Toledano and Alvarez 2004, 2010; Swarowsky et al., 2008; Leung et al., 2011), or the rat models of other deep brain structure lesions (Rugg et al., 1992; Inglis and Semba, 1997), even in the studies of the glial reactivity (Monzon-Mayor et al., 2000; Swarowsky et al., 2008), usually used $0.5-1 \mu \mathrm{L}$ microinfusion volume, that are much higher volumes then the highest volume used in our study. However, the impact of different volumes of excitotoxin or sham control solution was never tested. In contrast to other studies, we also compared the astrocytes responses with respect to mechanical lesion control. Our results have shown the suppressed reactive astroglial response to higher volumes of excitotoxin or sham control microinfusions versus mechanical lesion, 7 days after microinfusions, and only locally at the tip of microinfusion needle within NB. Although in our study we achieved the selectivity and completeness of the NB lesion with $200 \mathrm{~nL}$ and $100 \mathrm{~nL}$ of IBO microinfusion, to establish the rat model of $\mathrm{AD}$, for detailed analysis of the local, remote or proximal astrocytes responses, particularly induced by NB cholinergic neuronal damage (the cholinergic involution related glial response), we have to consider, beside excitotoxicity, an impact of the high microinfusion volume pressure within the local tissue that suppresses astrocyte reactivity. Regarding an importance of healthy astrocytes reactivity in all physiological and pathophysiological processes within the brain, and the possible consequences of their dysfunction, such as the reduced glutamate uptake and conversion of glutamate into glutamine via glutamine synthase (as an exclusive ability of astrocytes), our present study suggests only microinfusion volume less than $50 \mathrm{~nL}$ as protective for physiological astroglial reactivity in the in vivo animal models.

\section{Acknowledgements}

This work was supported by Serbian Ministry of Education, Science and Technological

Development, Grant OI 173022.

\section{References}

Anezaki T., Yanagisawa H., Takahashi T., Nakajima K., Miyashita K., Ishikawa A., Ikuta F., Miyatake $\mathrm{T}$. Remote astrocytic response of prefrontal cortex is caused by the lesions in the nucleus basalis of Meynert, but not in the ventral tegmental area. Brain Res., 574: 63-69, 1992.

Arendash G.W., Millard W.J., Dunn A.J., Meyer E.M. Long-term neuropathological and neurochemical effects of nucleus basalis lesions in the rat. Science, 238: 952-956, 1987.

Bignami D.D. Gliosis. In: Kettenmann H. and Ransom B.R. (Eds.). Neuroglia. New York, Oxford, Oxford University Press, 1995, pp. 843-858.

Datta S., Spoley E.E., Patterson E.H. Microinjection of glutamate into the pedunculopontine tegmentum induces REM sleep and wakefulness in the rat. Am. J. Physiol., 280: R752-759, 2001.

Dawson T.M., Bredt D.S., Fotuhi M., Hwang P.M., Snyder S.H. Nitric oxide synthase and neuronal NADPH diaphorase are identical in brain and peripheral tissues. Proc. Natl. Acad. Sci. USA, 88: 7797-7801, 1991.

Eng L.F. and Ghirnikar R.S. GFAP and astrogliosis. Brain Pathol., 4: 229-237, 1994.

Fulop Z.L., Lescaudron L., Geller H.M., Sutton R., Stein D.G. Astrocytes grafted into nucleus basalis magnocellularis immediately after ibotenic acid injection fail to survive and have no effect on functional recovery. Int. J. Neurosci., 90: 203-222, 1997.

Hope B.T., Gregory M.J., Knigge K.M., Vincent S.T. Neuronal NADPH diaphorase is a nitric oxide synthase. Proc. Natl. Acad. Sci. USA, 88: 2811-2814, 1991.

Inglis W.L. and Semba K. Discriminable excitotoxic effects of ibotenic acid, AMPA, NMDA and quinolinic acid in the rat laterodorsal tegmental nucleus. Brain Res., 755: 17-27, 1997.

Leung L.S., Petropoulos S., Shen B., Luo T., Herrick I., Rajakumar N., Ma J. Lesion of cholinergic neurons in nucleus basalis enhances response to general anesthetics. Exp. Neurol., 228: 259-269, 2011.

Lu J., Sherman D., Devor M., Saper C.B. A putative flip-flop switch for control of REM sleep. Nature, 441: 589-594, 2006.

McKay L.C., Janczewski W.A., Feldman J.L. Sleepdisordred breathing after targeted ablation of preBötzinger complex neurons. Nat. Neurosci., 8: 1142-1144, 2005.

Monnier A., Alheid G.F., McCrimmon D.R. Defining ventral medullary respiratory compartments with a glutamate receptor agonist in the rat. J. Physiol. (London), 548: 859-874, 2003.

Monzon-Mayor M., Alvarez M.I., Arbelo-Galvan J.F., Romero-Aleman M.M., Yanes C., Plaza 
M.L., Rodriguez J.R., Rodriguez J.J., Toledano A. Long-term evolution of local, proximal and remote astrocyte responses after diverse nucleus basalis lesioning (an experimental Alzheimer model): GFAP immunocytochemical study. Brain Res., 865: 245-258, 2000.

Nicholson C. Diffusion from an injected volume of a substance in brain tissue with arbitrary volume fraction and tortuosity. Brain Res., 333: 325-329, 1985.

Paxinos G., Watson C. The Rat Brain in Stereotaxic Coordinates. Sydney/New York, Academic Press, 2004.

Rugg E.L., Dunbar J.S., Latimer M., Winn P. Excitotoxic lesions of the pedunculopontine tegmental nucleus of the rat. I Comparison of the effects of various excitotoxins with particular reference to the loss of immunohistochemically identified cholinergic neurons. Brain Res., 589: 181-193, 1992.

Sabbatini M., Bronzetti E., Felici L., Fringuelli Ch., Sagratella S., Amenta F. NADPH-diaphorase histochemistry in the rat cerebral cortex and hippocampus: effect of electrolytic lesions of the nucleus basalis magnocellularis. Mech. Aging Dev., 107: 147-157, 1999.

Saponjic J., Cvorovic J., Radulovacki M., Carley D.W. Serotonin and noradrenaline modulate respiratory pattern disturbance evoked by glutamate injection into pedunculopontine tegmentum of anesthetized rats. Sleep, 28: 560-570, 2005a.

Saponjic J., Radulovacki M., Carley D.W. Respiratory pattern modulation by the pedunculopontine tegmental nucleus. Respir. Physiol. Neurobiol., 138: 223-237, 2003.
Saponjic J., Radulovacki M., Carley D.W. Injection of glutamate into the pedunculopontine tegmental nuclei of anesthetized rat causes respiratory dysrhythmia and alters EEG and EMG power. Sleep Breath., 9: 82-91, 2005b.

Swarowsky A., Rodrigues L., Biasibetti R., Leite M.C., de Oliveira L.F., de Almeida L.M.V., Gottfried C., Quillfeldt J.A., Achaval M., Goncalves C.A. Glial alteration in the hippocampus of rats submitted to ibotenic-induced lesion of the nucleus basalis magnocellularis. Behav. Brain Res., 190: 206-211, 2008.

Toledano A. and Alvarez M.I. Lesions and dysfunctions of the nucleus basalis as Alzheimer's disease models: general and critical overview and analysis of the long-term changes in several excitotoxic models. Curr. Alzheimer Res., 1: 189214, 2004

Toledano A. and Alvarez M.I. Lesion induced vertebrate models of Alzheimer Dementia. In: De Deyen P.P. and Van Dam D. (Eds.) Animal Models of Dementia. New York, Humana Press, 2010, pp. 295-345.

Topchiy I., Waxman J., Radulovacki M., Carley D.W. Functional topography of respiratory, cardiovascular and pontine-wave responses to glutamate microstimulation of the pedunculopontine tegmentum of the rat. Respir. Physiol. Neurobiol., 173: 64-70, 2010.

Vincent S.R., Satoh K., Armstrong D.M., Fibiger H.C. NADPH-diaphorase: a selective histochemical marker for the cholinergic neurons of the pontine reticular formation. Neurosci. Lett., 143: 31-36, 1983. 